\title{
La revolución brousseauniana como razón de ser del grupo Didáctica de las Matemáticas como Disciplina Científica ${ }^{1}$
}

\author{
Josep Gascón, Universitat Autònoma de Barcelona (España)
}

Recibido el 9 de Noviembre de 2012; aceptado el 1 de Febrero de 2013

\begin{abstract}
La revolución brousseauniana como razón de ser del grupo Didáctica de las Matemáticas como Disciplina Científica

\section{Resumen}

El grupo de trabajo "Didáctica de la Matemática como Disciplina Cientifica" (DMDC), heredero del Seminario Interuniversitario de Investigación en Didáctica de las Matemáticas (SIIDM), surgió con la intención de contribuir al desarrollo de la didáctica en la dirección propuesta por Guy Brousseau en la década de los 80. En particular, la ambición de construir una ciencia didáctica caracterizada como epistemología experimental, la transformación y ampliación de su objeto de estudio y la consiguiente inauguración del programa epistemológico de investigación en didáctica de las matemáticas constituyen, aún hoy día, la razón de ser del grupo DMDC.
\end{abstract}

Palabras clave. Ciencia didáctica, teoría de las situaciones didácticas, epistemología experimental, programa epistemológico.

The Brousseaunian revolution as the raison d'être of the group Didactics of Mathematics as a Scientific Discipline

\section{Abstract}

The group "Didactics of Mathematics as a Scientific Discipline” (DMDC), heir of the “

Interuniversity Seminar of Research in Didactics of Mathematics" (SIIDM), emerged with the aim of development of didactics in the direction proposed by Guy Brousseau in the 80s. More precisely, the ambition to build on a science of didactics characterised as an experimental epistemology, the transformation and enlargement of its object of study and the inauguration of the epistemological programme in didactics of mathematics represent the main rationale of the DMDC group.

Key words. Science of didactics, theory of didactic situations, experimental epistemology, epistemological programme.

\footnotetext{
${ }^{1}$ El grupo Didáctica de las Matemáticas como Disciplina Científica (DMDC) es un grupo de investigación integrado en la Sociedad Española de Investigación en Educación Matemática (SEIEM). Una versión simplificada de este trabajo fue presentada en el XVI Simposio de la SEIEM como Seminrio de investigación: Aportaciones a la Investigación desde la Didáctica de la Matemática como Disciplina Científica. Baeza, 20-22 Septiembre 2012 (Cf. Gascón 2012).
}

Para citar: Gascón (2013). La revolución brousseauniana como razón de ser del grupo Didáctica de las Matemáticas como Disciplina Científica. Avances de Investigación en Educación Matemática, 3, 69-87

(C) Sociedad Española de Investigación en Educación Matemática (SEIEM). www.seiem.es 
A revolução brousseauniana como razão de ser do grupo Didática da Matemática como uma Disciplina Científica

\title{
Resumo
}

O grupo de trabalho "Didática da Matemática como uma Disciplina Científica” (DMDC), herdeiro do Seminário Interuniversitário de Investigação em Didática da Matemática (SIIDM), surgiu com o intuito de contribuir para o desenvolvimento da didática na direção proposta por Guy Brousseau na década de 80. Em particular, a ambição de construir uma ciência didática caracterizada como "epistemologia experimental", a transformação e a ampliação do seu objeto de estudo e a consequente inauguração do programa epistemológico de investigação em didática da matemática constituem, ainda hoje em dia, a razão de ser do grupo DMDC.

Palavras chave. Ciência didática, teoria das situações didáticas, epistemologia experimental, programa epistemológico.

La révolution brousseaunienne comme raison d'être du groupe Didactique des Mathématiques comme Discipline Scientifique

\section{Résumé}

Le groupe de travail "Didactique des mathématiques comme discipline scientifique (DMDC)", héritier du Séminaire interuniversitaire de recherche en didactique des mathématiques (SIIDM) est apparu avec l'intention de contribuer au développement de la didactique des mathématiques dans la voie proposée par Guy Brousseau dans les années 80. Tout particulièrement, l'ambition de construire une science didactique caractérisée comme "épistémologie expérimentale", la transformation et élargissement de son objet d'étude et l'inauguration du programme épistémologique de recherche en didactique des mathématiques constituent, aujourd'hui, la raison d'être du groupe DMDC.

Paroles clés. Science didactique, théorie des situations didactiques, épistémologie expérimentale, programme épistémologique

\begin{abstract}
A este instrumento de explicación de las matemáticas y de su génesis lo llamo didáctica fundamental. Esto es una toma de posición teórica y supone que la teoría didáctica puede revolucionar la epistemología y transformar la descripción de la construcción de un conocimiento [matemático] [...]. Es un desafío, pero se puede comprender que este desafío está ligado a la ambición de construir la didáctica.
\end{abstract}

Guy Brousseau (1996, p. 40)

\section{Introducción}

En este trabajo presentaré una interpretación de la historia de una pequeña comunidad de investigadores en didáctica de las matemáticas que, en la actualidad, constituye uno de los grupos de la SEIEM, el denominado Didáctica de las Matemáticas como Disciplina Científica (DMDC). Se trata de una comunidad que inició su camino en 1991 bajo las siglas de Seminario Interuniversitario de Investigación en Didáctica de las Matemáticas (SIIDM) y cuya evolución dio lugar en 1998 al grupo DMDC.

Los investigadores que formamos parte de este grupo seguimos compartiendo, en lo esencial y con los matices y las variaciones que proporcionan los diferentes enfoques e intereses científicos, una manera de interpretar la didáctica que está inspirada, en última instancia, en lo que denomino "revolución brousseauniana" de la didáctica de las matemáticas. Es por ello que considero dicha revolución, o mejor, 
algunos de los supuestos básicos de la misma, como la "razón del ser" del grupo DMDC.

Pasados más de 25 años desde la publicación, en 1986, de los Fondements de Guy Brousseau, creo que es pertinente discutir el alcance de algunas de las ideas básicas de la teoría de las situaciones didácticas (TSD). Mi aportación a dicha discusión consistirá en proponer una interpretación poco habitual de la génesis y el desarrollo de dicha teoría ${ }^{2}$. De todas formas debo subrayar con toda claridad que no pretendo, de ninguna manera, hacer una descripción alternativa global de la TSD, únicamente me ocuparé de algunos rasgos originales de dicha teoría y de su incidencia sobre la creación del SIIDM y los desarrollos posteriores del mismo.

En lo que sigue, y después de presentar un breve esbozo de las etapas del desarrollo histórico del SIIDM y de su heredero el grupo DMDC, me centraré en analizar algunos de los citados rasgos que caracterizan la revolución brousseauniana.

Empezaré explicando la importancia que tuvo y sigue teniendo la ambición original de la TSD de construir una ciencia didáctica relativamente autónoma, con un objeto de estudio propio y en la que la noción de "fenómeno didáctico" ocupa una posición central.

Postularé que, después de la ruptura que provocó el programa cognitivo, la TSD, al tomar la actividad matemática como el objeto primario de estudio y como nueva "puerta de entrada" del análisis didáctico, inauguró un nuevo programa de investigación en didáctica de las matemáticas, el programa epistemológico.

Mostraré que la TSD invierte el objeto de estudio que proponía la didáctica clásica en el sentido de Brousseau (1986) y que esta inversión requiere cuestionar las formas habituales de describir e interpretar los "conocimientos matemáticos". Expondré, finalmente, cómo la TSD pone de manifiesto la necesidad de construir modelos epistemológicos propios (de los diferentes ámbitos de las matemáticas) para abordar los problemas didácticos en los que dichos conocimientos están involucrados. Este rasgo que, en cierto sentido contiene a los dos anteriores, es probablemente el más importante y el que caracteriza el nacimiento de la didáctica de las matemáticas como epistemología experimental.

En coherencia con lo anterior, propongo caracterizar los enfoques o teorías didácticas que forman parte del programa epistemológico como aquellos que cuestionan los modelos epistemológicos de las matemáticas dominantes en las diversas instituciones (por ejemplo, las instituciones escolares) y, lo que es más importante, como aquellos que elaboran explícitamente modelos epistemológicos alternativos de los diferentes ámbitos de las matemáticas y los utilizan como sistema de referencia para formular y abordar los problemas didácticos. Con esta caracterización no pretendo, por supuesto, hacer una partición en dos clases disjuntas en el extenso y complejo conjunto de enfoques y teorías didácticas que subsisten en la actualidad. Trato únicamente de explicitar un "criterio de demarcación" relativo y provisional que pone el acento en una característica que considero esencial en los enfoques del programa epistemológico y que, aún siendo una cuestión de grado (puesto que algunas teorías didácticas utilizan de manera más o menos implícita los

\footnotetext{
${ }^{2}$ Quiero agradecer la generosidad de Guy Brousseau que leyó una versión provisional de este trabajo y se mostró completamente de acuerdo con la interpretación que aquí se hace de algunos de los supuestos básicos de la TSD. Obviamente el único responsable de las opiniones que se vierten en esta versión definitiva del trabajo es el autor del mismo.
} 
citados modelos epistemológicos alternativos), puede ser útil para orientarnos en relación al tipo de problemas didácticos que plantean y abordan los diversos enfoques y, también, en relación a lo que se considera en cada caso como una respuesta aceptable a dichos problemas.

\section{Breve historia de una comunidad de investigación: del SIIDM al DMDC}

La comunidad de investigación que sustenta el grupo Didáctica de las Matemáticas como Disciplina Científica (DMDC), heredero del Seminario Interuniversitario de Investigación en Didáctica de las Matemáticas (SIIDM), tiene una historia de más de 20 años (1991-2012). En esta historia podemos distinguir tres etapas:

1991-1997: En esta primera etapa sólo existía el SIIDM y en ella se celebraron un total de 11 Seminarios en el Departamento de Didáctica de la Matemática de la Universidad Complutense de Madrid (UCM).

1998-2004: La segunda etapa se inicia con la celebración en 1998 del XII SIIDM en Baeza y con la creación ese mismo año del grupo DMDC como grupo de trabajo de la Sociedad Española de Investigación en Educación Matemática (SEIEM). Los posteriores Seminarios se celebraron en El Escorial (1999), Cangas do Morrazo (2000), Huelva (2000), Huesca (2001), Almería (2001), Castellón (2002), Córdoba (2003) y Madrid (2004). En esta etapa convivieron el SIIDM y el DMDC.

2005-2012: A partir de $2005^{3}$ el SIIDM se diluyó definitivamente dentro del grupo DMDC y se inició una nueva etapa a lo largo de la cual los intereses de los miembros del grupo han ido evolucionando hasta confluir, principalmente, en dos potentes subgrupos que trabajan respectivamente en el enfoque ontosemiótico (EOS) y en la teoría antropológica de lo didáctico (TAD).

A lo largo de las 20 reuniones del SIIDM (celebradas entre 1991 y 2004) se presentaron y discutieron una gran cantidad de comunicaciones sustentadas en los diversos enfoques y marcos teóricos que han convivido en el seno del SIIDM y del grupo DMDC, tal como muestra el trabajo que presentamos en Huelva en el marco del IV Simposio de la SEIEM (Ruiz Higueras et al. 2001). Una información bastante completa de estos trabajos se encuentra en el sitio http://www.ugr.es/ jgodino//siidm/ y, más concretamente, en los boletines del SIIDM. Por otra parte, las contribuciones presentadas dentro del grupo DMDC entre 2005 y 2012 están publicadas en las actas de los Simposios de la SEIEM ${ }^{4}$.

\footnotetext{
${ }^{3}$ En el año 2005 se celebró, también en Baeza, el primer congreso internacional sobre la teoría antropológica de lo didáctico (I CITAD) con motivo de los 25 años de la primera publicación de Yves Chevallard, en 1980, sobre la teoría de la transposición didáctica, germen de la TAD. Los trabajos presentados en el I CITAD están publicados en Ruiz-Higueras, Orús, Godino, y Gascón (2007). Los sucesivos CITAD se han celebrado en Uzès (Bronner, Larguier, Artaud, Bosch, Chevallard, Cirade, \& Ladage, 2010) y en Sant Hilari Sacalm (Bosch et al. 2011). Todos estos documentos son accesibles en la página web del grupo TAD $\underline{w w w . a t d-t a d . o r g}$

${ }^{4}$ No entraré aquí a analizar el contenido de los trabajos que hemos desarrollado a lo largo de estos 21 años de historia. De hecho en el mismo XVI Simposio de la SEIEM y dentro del Seminario de Investigación Aportaciones a la Investigación desde la Didáctica de la Matemática como Disciplina Científica se han presentado dos trabajos que describen con cierto detalle una parte importante de las aportaciones del grupo DMDC a la investigación didáctica (ver Godino, 2012 y Bosch, 2012).
} 
La actual consolidación de los enfoques y teorías didácticas que conviven en el seno del grupo DMDC abre una nueva etapa en el desarrollo del grupo. En mi opinión se trata de una etapa en la que el diálogo entre los enfoques teóricos que conviven dentro del grupo y entre estos y los restantes enfoques del continente didáctico ocupará un lugar central. Después de un periodo de relativo "aislamiento", que podría interpretarse como una reclusión voluntaria de las comunidades de didactas para cohesionarse y poder cuestionar libremente la visión cultural de su ámbito de estudio (esto es, las nociones culturales y las ideas socialmente dominantes sobre "lo matemático" y "lo didáctico") y que, no por casualidad, ha coincidido con la etapa fundacional de la ciencia didáctica, ha llegado el momento de contrastar, mediante el diálogo científico, las diferentes teorías didácticas.

De hecho, la etapa de diálogo entre enfoques ya se ha iniciado. Entre los muchos trabajos en los que se lleva a cabo algún tipo de diálogo entre diferentes teorías didácticas, y en los que está involucrado alguno de los enfoques que conviven dentro el grupo DMDC, podemos citar los siguientes: Wilhelmi, Font, y Godino (2005); Bolea, Bosch, García, Gascón, Ruiz Higueras, y Sierra (2005); Godino, Font, Contreras, y Wilhelmi (2006); D'Amore y Godino (2007); Font, Godino, y D'Amore (2007); Prediguer, Arzarello, Bosch, y Lenfant (2008); Rodríguez, Bosch, y Gascón (2008); Arzarello, Bosch, Gascón, y Sabena (2008); Bikner-Ahsbahs, Dreyfus, Kidron, Arzarello, Radford, Artigue, y Sabena (2010); García y Wake (2010); Artigue, Bosch, Gascón, y Lenfant (2010); García y Ruiz-Higueras (2010 y 2011); Trigueros, Bosch, y Gascón (2011); Artigue, Bosch, y Gascón (2011a y 2011b); RuizMunzón, Matheron, Bosch, y Gascón (2012); Drijvers, Godino, Font, y Trouche (2012) y Sierra, Bosch, y Gascón (2012).

Observamos que la necesidad de diálogo entre los diversos enfoques o teorías didácticas se ha acrecentado en esta última década y postulamos un fuerte desarrollo de esta línea de investigación en los próximos años. Uno de los diálogos pendientes es el que debe llevarse a cabo entre el EOS y la TAD que, como he dicho, constituyen los dos principales enfoques que coexisten actualmente dentro del grupo DMDC.

\section{La revolución brousseauniana de la didáctica de las matemáticas}

En 2003, Guy Brousseau recibió la primera medalla Félix Klein otorgada por la Comisión Internacional de Instrucción Matemática (ICMI) como reconocimiento a una vida dedicada a la construcción de una gran obra científica: la teoría de las situaciones didácticas (TSD). Aunque algunas de las interpretaciones habituales de la TSD tienden a hacerla compatible con los enfoques comúnmente aceptados, mostraré que, en realidad, la TSD inaugura un nuevo programa de investigación (en el sentido de Lakatos, 1978) en didáctica de las matemáticas, el que he denominado programa epistemológico (Gascón, 1998 y 2003).

En los trabajos germinales de la TSD, publicados durante la década de los 80 del siglo pasado, Guy Brousseau propuso una nueva y revolucionaria interpretación de la problemática didáctica que fue el acicate que impulsó la constitución de nuestra pequeña comunidad científica. Además, el estudio y desarrollo de dicha problemática constituye aún actualmente la "razón de ser" de nuestro grupo, esto es, lo que justifica nuestra forma de interpretar y abordar los fenómenos didácticos.

A fin de contextualizar históricamente la relación entre el origen del SIIDM y los postulados iniciales de la TSD, empezaré citando los puntos más significativos del 
documento constitutivo del SIIDM que aparece en el boletín $\mathrm{n}^{\circ} 1$ de dicho seminario (1991) (ver http://www.ugr.es/ jgodino//siidm/).

Los miembros del Seminario comparten un interés particular por el marco teórico de la didáctica de las matemáticas iniciado por el profesor Guy Brousseau sin que ello suponga el cierre a otras perspectivas que puedan servir de fundamentación para la didáctica de las matemáticas.

El principal objetivo del Seminario es el de profundizar en el estudio y comprensión de los fenómenos didácticos, es decir, de aquellos fenómenos relacionados con la producción y comunicación del conocimiento matemático.

En este punto es importante destacar la mención que se hace a los "fenómenos didácticos" como objeto primario de estudio de la didáctica de las matemáticas y, también, enfatizar que los fenómenos relacionados con la "producción del conocimiento matemático" se integran en dicho objeto de estudio. Esta ampliación del objeto de estudio de la didáctica clásica constituye uno de los postulados o asunciones básicos que sustentaron la creación del SIIDM.

En lo que sigue no pretendo hacer una descripción objetiva e imparcial de la creación del SIIDM porque esta sería una pretensión ilusoria y engañosa. Únicamente expondré mi interpretación de las ideas que impulsaron el nacimiento de dicho seminario.

\subsection{La ambición de construir una ciencia didáctica con un objeto de estudio propio. La noción de fenómeno didáctico}

La TSD construye por primera vez la noción de fenómeno didáctico, entendido inicialmente como una regularidad no intencional que no se puede reducir a fenómenos cognitivos, sociológicos o lingüísticos. La didáctica de las matemáticas construye los fenómenos didáctico-matemáticos (por ejemplo, el "efecto Topaze", el "deslizamiento meta-didáctico" o la "aritmetización del álgebra escolar") para explicar los hechos didácticos (empíricos) de manera racional y para relacionarlos con otros fenómenos.

En los Fondements de Brousseau, publicados en 1986, se postula que los fenómenos didácticos, esto es, los fenómenos que emergen en las actividades de producción y difusión de las matemáticas, constituyen el objeto primario de investigación de la didáctica de las matemáticas. Cinco años más tarde, en 1991, el documento fundacional del SIIDM muestra que esta forma de interpretar la didáctica es compartida por los miembros de nuestra incipiente comunidad. Es importante subrayar que, en ese momento, la ambición de construir una ciencia de los fenómenos didácticos estaba prácticamente ausente de la comunidad de Educación Matemática y que, incluso actualmente, podemos constatar que ni la noción de fenómeno didáctico ni su relación con los problemas de investigación didáctica juegan un papel central en la mayoría de los enfoques en didáctica de las matemáticas. Tal como hemos señalado en Artigue, Bosch, y Gascón (2011b, p. 2383):

Today the notion of 'phenomenon' does not happen to have a central function in many didactics of mathematics approaches. It did however play a crucial role in the emergence of the theory of didactic situations (TDS) and its vision of didactics as a scientific discipline. In the first developments of this approach in the 1980s, and through several different formulations, Brousseau (1997) defined didactics of mathematics as the science the essential aim of which is the knowledge of didactic phenomena, that is, phenomena appearing in the teaching, learning or, more generally, 
diffusing of mathematical knowledge in social institutions (including school ones). Hence, didactic phenomena have to be considered both as a construction and as an object of study of didactics, in the same way that physics studies the specific construction 'physical phenomena', or sociology studies and also defines social phenomena, etc. -including all the historical controversies about phenomena delimitation in nature and social sciences.

Y en este mismo trabajo se enfatiza la importante relación entre los fenómenos didácticos y los problemas de investigación didáctica (Ibíd, p. 2384):

As any scientific discipline, didactics of mathematics aims at identifying and studying a specific kind of phenomena (didactic ones) in order to attain a greater capacity of action and comprehension. Any research question or problem thus has to be related - even if this relation is mostly done a posteriori - to the highlighting of a phenomenon, its delimitation, the conditions needed for its existence and evolution, etc. Even if research takes as a starting point a problem emerging from a very concrete teaching or learning practice (as it often does), an effort is always made to formulate the problem in a more general way, implicitly considering it as a specimen of a given 'type of problem', which is a first step in the work that follows: looking for regularities related to the practical issue approached, trying to characterise them and, to some extent, 'understand' or 'act upon' them. Let us consider, for instance, the phenomenon of didactic transposition as it was characterised by Yves Chevallard (1985). Several new problems have been raised and studied that could not even have been formulated before the identification of the phenomenon (see Bosch y Gascón 2006 for a recent review).

Para recuperar el papel central que, en mi opinión, deberían tener los fenómenos didáctico-matemáticos en el proceso de construcción de la problemática didáctica, he propuesto, en Gascón (2011), interpretarlos como "fenómenos de la vida social" que se construyen utilizando "conceptos-tipo ideales" en el sentido de Max Weber $(1904 / 2009)^{5}$.

Max Weber afirma que las ciencias sociales tienen por objeto los "fenómenos de la vida social" que, añade, pueden ser considerados como "individuos históricos" en el sentido que evolucionan históricamente junto a la cultura y al cambio de valores sociales. Cita, como ejemplos, la "ética calvinista", el "espíritu capitalista", el "feudalismo occidental", el "artesanado" y el "imperialismo", entre otros. Todo concepto-tipo ideal se construye como concepto abstracto de un fenómeno que nos representamos (mediante una conjetura) como realidad persistente en el flujo del acontecer y que, postulamos, será útil para analizar un conjunto de hechos empíricos, para explicarlos de manera racional y para relacionarlos con otros fenómenos.

Weber postula que toda disciplina científica tiene necesidad de construir "tipos ideales" de conceptos que funcionan como "conceptos límites" o "conceptos ideales". Estos tipos ideales están caracterizados teóricamente pero no son copias fotográficas de los hechos empíricos, esto es, no existen en estado puro en la realidad histórica. Podemos interpretarlos como modelos y, como tales, tienen una función metodológica, heurística, puesto que sirven para compararlos (o contrastarlos) con la realidad empírica, históricamente existente. Como en todo proceso de modelización científica es importante no confundir el sistema empírico modelizado, que siempre es infinitamente complejo, con el modelo cuya función no es copiar el sistema sino producir conocimientos sobre el mismo. En este sentido la construcción de los "tipos ideales" y su contrastación empírica forma parte fundamental de las técnicas de investigación científica.

En el caso de la didáctica de las matemáticas podemos citar multitud de tipos ideales de conceptos tales como: el "contrato didáctico", la "transposición didáctica",

\footnotetext{
${ }^{5}$ El adjetivo "ideal" no indica ningún tipo de perfección, esencialidad ni valoración, tiene que ver exclusivamente con la función puramente lógica, de modelo, que el "tipo ideal” cumple.
} 
la "noosfera", el "efecto Topaze", el "deslizamiento metadidáctico" la "obsolescencia de las situaciones de enseñanza" y la "aritmetización del álgebra escolar", entre otros muchos. Cuando hablamos de cualquiera de estos tipos ideales, nos referimos por una parte al sistema empírico históricamente existente (conjunto de hechos empíricos no muy bien delimitados y que nunca pueden conocerse perfectamente ni describirse de manera exhaustiva) y, por otra, al modelo construido con relativa precisión por la disciplina didáctica de las matemáticas. Es precisamente la comparación entre el tipo ideal (el modelo construido por la didáctica) y la realidad empírica (la contingencia histórica), la que proporciona la posibilidad de formular hipótesis explicativas relativas a los hechos empíricos y a su relación con otros fenómenos.

¿Cómo se construyen estos tipos ideales de conceptos? A partir de un conjunto de hechos históricos (en nuestro caso "hechos didácticos", en el sentido de hechos relativos a la génesis y difusión del conocimiento matemático) aparentemente desligados entre sí, el investigador, utilizando las herramientas teóricas y metodológicas que le proporciona una disciplina científica, elige algunas características que comparten en mayor o menor medida los hechos citados, acentúa algunas de ellos y hasta incorpora otras características que sólo están presentes de manera excepcional (e incluso incluye algunas que pueden estar ausentes) en el conjunto de hechos de partida. De esta manera, después de postular algunas relaciones entre las características seleccionadas, el investigador construye teóricamente un "concepto-tipo ideal" que permite describir ciertos fenómenos. Es de esta forma como se ha construido, por ejemplo, la noción de "contrato didáctico" que es un importante "concepto-tipo ideal" y, como tal, permite describir e interpretar muchos hechos didácticos y establecer relaciones con otros fenómenos.

\subsection{Del programa cognitivo al programa epistemológico de investigación en didáctica de las matemáticas ${ }^{6}$}

¿En qué sentido la TSD transforma de manera radical el objeto de estudio de la didáctica? ¿Cómo se construye y cuál es la amplitud del objeto de estudio renovado? Para responder a estas cuestiones utilizaré, de forma simplificada, un esquema propuesto en Gascón (1998). Con ayuda de este esquema podemos decir que, una vez establecido que la respuesta pedagógica al problema de la Educación Matemática es claramente insuficiente, se produjo la ruptura con la Pedagogía. Esta ruptura fue obra del programa cognitivo de investigación en didáctica de las matemáticas (Gascón, 2002 y 2003).

El acta de nacimiento del programa cognitivo de investigación en didáctica de las matemáticas puede situarse en el influyente trabajo de Bauersfeld y Skowronek (1976) en el que reivindicaban explícitamente la necesidad de tomar en consideración la construcción de una teoría del aprendizaje específicamente matemático, en lugar de empezar por una teoría del aprendizaje general. Una propuesta similar, de ampliación de lo pedagógico-cognitivo mediante la introducción de componentes "disciplinares" (en nuestro caso componentes "matemáticos") fue defendida, en el ámbito de la formación del profesorado, por Lee Shulman $(1986,1987)$ mediante la noción de conocimiento pedagógico del contenido (pedagogical content knowledge, PCK).

\footnotetext{
${ }^{6}$ En este trabajo no analizaremos la incidencia que ha tenido la transformación del objeto de estudio de la didáctica sobre las metodologías de investigación (Cf. Godino et al. 2012).
} 
El programa cognitivo cuestionó la presunta suficiencia del enfoque pedagógico y de la noción de "aprendizaje humano" para abordar los problemas matemáticodidácticos y postuló la necesidad de modelizar el "aprendizaje matemático (del alumno)", lo que comportó, de hecho, una ampliación de lo "pedagógico-cognitivo" para incluir componentes "matemáticos" $\mathrm{y}$, consecuentemente, una primera ampliación del objeto de estudio de la didáctica.

En el marco del programa cognitivo el objeto primario de investigación está constituido por los procesos cognitivos $^{7}$ que intervienen en las situaciones de enseñanza-aprendizaje matemático que protagonizan los sujetos. Este aprendizaje se supone fuertemente condicionado por la actividad docente del profesor al que se le considera como un mediador directo e inmediato entre la matemática a enseñar y los alumnos. Estos presupuestos comportan que el conocimiento matemático que entra en juego se suponga dado, transparente, incuestionable y, en definitiva, no problemático y, además, que se tienda a asignar a dicho conocimiento un cierto carácter "absoluto", ignorándose así, en cierta forma, la relatividad institucional de los conocimientos matemáticos.

En estas condiciones no es fácil cuestionar el modelo epistemológico implícito dominante en una institución didáctica ni, por tanto, estudiar los fenómenos didácticomatemáticos que permanecen invisibles. De hecho, una característica común de las investigaciones didácticas desarrolladas en el ámbito del programa cognitivo consiste en asumir la interpretación escolar (o, en su caso, la interpretación de la comunidad matemática "sabia") de los conocimiento matemáticos involucrados en la misma. Un ejemplo paradigmático de esta asunción y de las limitaciones que comporta, nos lo proporciona el modelo epistemológico dominante del "álgebra elemental" en la enseñanza secundaria actual; este modelo identifica el "álgebra elemental" con una especie de "aritmética generalizada" y ha llevado a encerrar en el "marco de referencia aritmético" a la mayoría de investigaciones relativas a la enseñanza y el aprendizaje del álgebra elemental en Secundaria (Gascón, 1993, 1994, 1995 y 1999; Bolea, 2003; Bolea, Bosch, \& Gascón, 2001a, 2001b y 2004; Ruiz-Munzón, 2010; Ruiz-Munzón, Bosch, \& Gascón, 2010 y 2011; Ruiz-Munzón et al., 2012).

El programa epistemológico de investigación en didáctica de las matemáticas asume la ruptura con la Pedagogía y surge de la convicción de que el origen del problema de la Educación Matemática está en la forma de interpretar las propias matemáticas. $\mathrm{Y}$ al cuestionar la transparencia de lo que es considerado como "matemático" y tomar la actividad matemática como objeto primario de estudio, como nueva "puerta de entrada" del análisis didáctico, se produce una segunda ruptura con la didáctica clásica que se materializa en el cuestionamiento de los modelos epistemológicos habituales de los diferentes ámbitos de las matemáticas.

La emergencia del programa epistemológico provoca una nueva ampliación del objeto de estudio de la didáctica al hacerse visibles fenómenos didácticos que, o habían permanecido invisibles o no eran considerados como objetos de estudio de la didáctica. Entre dichos fenómenos podemos citar: el "carácter prealgebraico del currículo de la enseñanza obligatoria" (Bolea, Bosch y Gascón 2001a); la "irresponsabilidad matemática de los alumnos", la "paradoja de la creatividad

\footnotetext{
7 Según la psicología cognitiva, los "procesos cognitivos" son procesos mediante los cuales las personas adquieren, almacenan, recuperan y usan el conocimiento. El paradigma cognitivo emergió en psicología a mediados del siglo XX y su desarrollo fue, en primera instancia, una consecuencia de las insuficiencias explicativas del conductismo.
} 
matemática" y la "ausencia de la verdadera disciplina matemática en las instituciones escolares" (Chevallard, Bosch, \& Gascón, 1997) y la "algebrización del cálculo diferencial escolar" (Artigue, 1995).

Dichos fenómenos sólo pueden ser abordados científicamente si determinados objetos (como "actividad matemática creativa", "cálculo diferencial escolar", "disciplina matemática", "proceso de enseñanza", etc.) que funcionaban como transparentes (para-didácticos) en el programa cognitivo pasan a ser objetos de estudio en sí mismos, esto es, objetos didácticos (tematizados y modelizados por la didáctica de las matemáticas), integrantes de pleno derecho de la problemática didáctica (Gascón, 1998, p.17). En esto consiste la originalidad del programa epistemológico en didáctica de las matemáticas, en abrir una nueva vía de acceso al estudio de los fenómenos didácticos a través de la modelización explícita del saber matemático enseñado. Uno de los rasgos esenciales de este punto de vista consiste precisamente en tomar la actividad matemática en sí misma y en particular la actividad matemática escolar, como objeto primario de investigación. Este es el origen de la denominación de "epistemología experimental" que Brousseau dio inicialmente a la didáctica de las matemáticas. Se pone así de manifiesto (o se postula) que todo fenómeno relativo a la enseñanza y al aprendizaje de las matemáticas tiene un componente matemático esencial (Brousseau, 1994).

El paso del programa cognitivo al programa epistemológico constituye lo que Lakatos denomina un "cambio progresivo de problemática", con el consiguiente aumento del "poder heurístico" del nuevo programa de investigación. Este aumento viene corroborado por la aparición de nuevos tipos de problemas, de nuevas teorías auxiliares y con la anticipación de hechos y fenómenos nuevos.

En particular, surge por primera vez la noción de "fenómeno didácticomatemático" que no se puede reducir a los fenómenos psicológicos, sociológicos o lingüísticos asociados. Se contradice de esta forma uno de los postulados implícitos básicos del programa cognitivo en el que se presuponía que todo fenómeno relativo a la enseñanza-aprendizaje de las matemáticas era reductible en última instancia a determinados fenómenos psicolingüísticos y semióticos. Es por esta razón que, mientras en el marco del programa cognitivo se usan los hechos didácticomatemáticos para explicar ciertos fenómenos psicolingüísticos y semióticos, en el programa epistemológico, por el contrario, se utiliza el comportamiento psicolingüístico y semiótico de los sujetos para explicar los fenómenos didácticomatemáticos.

La emergencia de los fenómenos didácticos-matemáticos así interpretados es característica del programa epistemológico y comporta la consiguiente emergencia de los problemas didáctico-matemáticos dado que, en toda disciplina científica, cada tipo de problemas hace referencia a un (aspecto de un) fenómeno. El núcleo firme de este nuevo programa de investigación está constituido inicialmente por un modelo epistemológico general de la actividad matemática (escolar) y, correlativamente, por los modelos epistemológicos específicos de los diferentes "ámbitos" de dicha actividad. El modelo docente y, en particular, lo que se entienda por "enseñar y aprender matemáticas", se formulará entonces haciendo uso de los términos primitivos de los citados modelos epistemológicos. 
Históricamente el programa epistemológico fue inaugurado por la teoría de las situaciones didácticas $^{8}$. A medida que se iba desarrollando el programa se puso de manifiesto que no era posible interpretar adecuadamente la actividad matemática escolar sin tener en cuenta los fenómenos relacionados con la reconstrucción escolar de las matemáticas que tienen su origen en la propia institución productora del saber matemático. Aparecen así los fenómenos de transposición didáctica (Chevallard, 1985) y, como una consecuencia natural, la teoría antropológica de lo didáctico (TAD). En ésta se toma inicialmente como objeto primario de investigación la actividad matemática institucionalizada y, por tanto, debe explicitarse un modelo general de las matemáticas institucionalizadas (que incluya, como caso particular, la matemática escolar) y un modelo de las actividades matemáticas institucionales (que incluya, en particular, la enseñanza-aprendizaje escolar de las matemáticas).

En los últimos desarrollos de la teoría antropológica de lo didáctico, dichos modelos se articulan alrededor de la noción de praxeología (matemática y didáctica) y constituyen el núcleo firme de la teoría antropológica en su versión actual.

En definitiva, el programa epistemológico constituye una respuesta a la insuficiencia manifiesta de los modelos epistemológicos dominantes en las instituciones escolares (Gascón, 2001) para formular y abordar los problemas didáctico-matemáticos y, en particular, reacciona contra el reduccionismo del modelo popular de las matemáticas (Thurston, 1994) que, al limitar la actividad matemática a series del tipo: "definición-especulación-teorema-prueba", provoca una separación radical entre el "hacer" y el "enseñar-aprender" matemáticas.

En el programa epistemológico, por el contrario, se postula la inseparabilidad entre lo matemático y lo didáctico. En el caso particular de la TSD se postula que un conocimiento matemático está definido por las situaciones que lo determinan, esto es, por un conjunto de situaciones para las que dicho conocimiento es idóneo porque proporciona la solución óptima en el contexto de una institución determinada. Las situaciones contienen la "razón de ser" del conocimiento que definen, esto es, las cuestiones que le dan sentido, así como las restricciones que limitan su uso en una institución determinada y las aplicaciones potenciales del mismo. Así, el modelo epistemológico de las matemáticas en el que se sustenta la TSD unifica lo matemático y lo didáctico mediante una ampliación de lo que se considera como "matemático" en las instituciones escolares. Esto es, para la TSD, la "razón de ser" de los conocimientos matemáticos y las restricciones que limitan su uso en una institución determinada, junto a las aplicaciones de dichos conocimientos, constituyen componentes matemáticos de pleno derecho de los propios conocimientos matemáticos.

En consecuencia, y como ya se ha dicho, con esta segunda ruptura se produce una nueva ampliación del objeto de estudio de la didáctica pasando a incluir la actividad matemática escolar que, en consecuencia, debe ser modelizada ya que toda disciplina modeliza lo que cuestiona y lo que pretende explicar. Posteriormente, la evolución interna de la TSD la ha llevado a considerar de forma aún más comprensiva el objeto de estudio de la didáctica:

\footnotetext{
${ }^{8}$ Considero que los trabajos iniciales de Guy Brousseau constituyen el germen del programa epistemológico. En Brousseau (1997, en inglés y 1998, en francés) se encuentra una recopilación de sus trabajos publicados entre 1970 y 1990. La página http://guy-brousseau.com/ contiene gran parte de su producción científica.
} 
Definición. La didáctica de las matemáticas es la ciencia de las condiciones de creación y difusión de los conocimientos matemáticos. (Brousseau, 1996, p. 33).

Para Guy Brousseau la didáctica de las matemáticas es una ciencia de carácter experimental tal como se refleja muy claramente a lo largo de toda su obra. Ya en 1972 G. Brousseau fundó el COREM, Centro para la Observación e Investigación en Enseñanza de la Matemática, dentro del marco de la Universidad de Bordeaux y en torno a la escuela J. Michelet de Talence. En dicho Centro, que dirigió durante más de 25 años, llevó a cabo una ingente investigación fundamental y experimental ligada a la enseñanza efectiva de las matemáticas en el aula. Los numerosos recursos documentales producidos en el COREM han sido cedidos a la Universitat Jaume-I de Castelló, constituyendo el Centro de Recursos de Didáctica de las Matemáticas Guy Brousseau dirigido por Pilar Orús (ver http://www.imac.uji.es/CRDM/index.php).

Asimismo, para la TSD, la didáctica es una ciencia más explicativa que normativa: La teoría de las situaciones didácticas se presenta en la actualidad como un instrumento científico. [...] Si bien algunos resultados de investigación han sido tomados como nuevos métodos de enseñanza, no es mi intención hacer proselitismo en este sentido. (Brousseau, 2007, p. 12).

$\mathrm{Y}$ es una ciencia que, en cierto sentido, puede considerarse predictiva porque aspira a prever tanto las condiciones más idóneas para que un tipo de fenómenos matemático-didácticos emerjan en una institución determinada, como las consecuencias colaterales que surgirían de su implantación. También pretende anticipar las restricciones que aparecerían si se intentara modificar en una dirección determinada la organización didáctico-matemática existente en una institución.

El énfasis en el carácter científico-experimental de la didáctica junto a la transformación y ampliación de su objeto de estudio influyeron decisivamente en 1998 no sólo en la elección del nombre del grupo DMDC ${ }^{9}$ sino, sobre todo, en el espíritu fundacional del mismo.

\subsection{La didáctica de las matemáticas como epistemología experimental}

La TSD estudia las características de las "situaciones didácticas", esto es, los modelos de interacción de un sujeto genérico con cierto "medio". Una situación didáctica determina (modeliza) un conocimiento matemático dado, como el recurso óptimo del que dispone el sujeto para alcanzar o conservar en esta situación un estado favorable. En consecuencia, la TSD invierte el problema que planteaba la didáctica clásica.

Desde la perspectiva de la teoría de las situaciones, los alumnos se convierten en los reveladores de las características de las situaciones a las que reaccionan (es importante señalar esta inversión de posición con respecto a las aproximaciones de la psicología, donde las situaciones suelen estudiarse como dispositivos para revelar los conocimientos del alumno). (Brousseau, 2007, p. 24)

Esta inversión del objeto de estudio requiere cuestionar las formas habituales de describir e interpretar los "conocimientos matemáticos". La TSD cuestiona los modelos epistemológicos (de los diferentes ámbitos de las matemáticas) dominantes en las diversas instituciones y construye modelos propios para abordar los problemas

\footnotetext{
${ }^{9}$ Uno de los grupos de trabajo del ICME 8, celebrado en Sevilla en julio de 1996, el Working Group 25 (en el que, junto a Guy Brousseau, participamos Juan D. Godino y yo mismo) se denominó precisamente Didactics of Mathematics as a Scientific Discipline (Malara, 1997).
} 
didácticos en los que intervienen los conocimientos en cuestión. Así, por ejemplo, para formular con precisión y abordar problemas didácticos en los que están involucrados ciertos ámbitos de la actividad matemática, la TSD cuestiona los modelos epistemológicos relativos a los números decimales, al contar, a la medida de magnitudes, a la geometría o a la relación entre la estadística y la probabilidad. En cada caso elabora un modelo epistemológico específico del correspondiente ámbito de la actividad matemática formulado en términos de situaciones.

Nace así la TSD como una epistemología experimental (de las matemáticas) con la ambición de explicar la génesis, el desarrollo y la difusión de las matemáticas. En esto consiste, precisamente, el principio metodológico fundamental de la TSD: definir un "conocimiento matemático" mediante una "situación", esto es, por un autómata que modeliza los problemas que únicamente este conocimiento permite resolver de forma óptima (Brousseau, 1994).

La "epistemología experimental" asume implícitamente que los datos de la psicogénesis, completados con los que proporciona la historia de las matemáticas, son insuficientes para describir e interpretar la génesis y el desarrollo de los conocimientos matemáticos en las instituciones escolares. La base empírica para abordar el problema epistemológico debe incluir los "hechos" que se producen en las instituciones didácticas porque la epistemología deber dar cuenta de fenómenos que dependen esencialmente de la institución didáctica en el seno de la cual tiene lugar la denominada génesis "personal", el desarrollo y la difusión de los conocimientos matemáticos (Gascón, 2001).

Aparece así un nuevo modelo epistemológico general de las matemáticas que viene a sustituir a los modelos epistemológicos conceptualistas dominantes en las instituciones docentes y más allá. En la TSD la actividad matemática escolar es siempre una actividad en situación, aunque en esta teoría se habla de "conocimiento matemático" para referirse, por ejemplo, a los números decimales, no se consideran los conceptos aislados sino la actividad matemática como tal.

La TSD cambia las antiguas cuestiones que constituían el objeto primario de investigación de la Educación Matemática:

- ¿Cómo aprenden matemáticas los alumnos? ¿Qué dificultades ponen de manifiesto?

- ¿Mediante qué mecanismos o procesos cognitivos adquieren los conceptos matemáticos los alumnos (o cómo los construyen)?

- ¿Qué métodos son los más adecuados para enseñar dichos conceptos?

Substituyéndolas por otras cuestiones de diferente naturaleza:

- ¿Qué condiciones debe satisfacer una situación para poner en funcionamiento los conocimientos específicos que la propia situación modeliza?

- ¿Cuáles son los efectos previsibles de dicho funcionamiento sobre los protagonistas y sobre sus producciones (fenómenos didácticos)?

- ¿Qué juego debe jugar el sujeto para necesitar un conocimiento determinado?

La TSD transformó así un problema centrado en la enseñanza-aprendizaje en otro de epistemología experimental de las matemáticas. La ruptura con el enfoque cognitivo dominante, centrado en el sujeto que aprende y, después, en el que enseña, supone una audacia difícil de asumir aún hoy día. La didáctica clásica pretende 
demostrar que tal método de enseñanza es mejor que tal otro, pero nadie había pretendido hasta entonces que, para formular con precisión y estudiar los problemas didácticos, era esencial la redefinición del álgebra, de la geometría, de la estadística, etc., mediante modelos que difieren abiertamente de los modelos epistemológicos dominantes en las instituciones escolares y en la institución "sabia". El misterio, lo que debe ser explicado en primera instancia, pasa de los procesos de enseñanzaaprendizaje a la forma de interpretar el saber matemático. En la TSD el misterio está, en primer lugar, en las situaciones consideradas como modelos de los conocimientos matemáticos.

Para concluir, postulo que los citados rasgos característicos de la TSD: la ambición de construir una ciencia didáctica, la transformación y ampliación de su objeto de estudio y la consiguiente inauguración del programa epistemológico de investigación en didáctica de las matemáticas, constituyen, aún hoy día, la razón de ser del grupo de investigación DMDC, esto es, la justificación de nuestra manera de interpretar la ciencia didáctica.

\section{Referencias}

Artigue, M. (1995). La enseñanza de los principios del cálculo: problemas epistemológicos, cognitivos y didácticos. En M. Artigue, R. Douady, L. Moreno, \& P. Gómez (Eds.), Ingeniería didáctica en educación matemática (pp. 97-140). México: Grupo Editorial Iberoamérica.

Artigue, M., Bosch, M., \& Gascón, J. (2011a). La TAD face au problème de l'interaction entre cadre théoriques en didactique des mathématiques. En M. Bosch, J. Gascón, A. Ruiz Olarría, M. Artaud, A. Bronner, Y. Chevallard, G. Cirade, C. Ladage, \& M. Larguier (Eds.), Un panorama de la TAD (vol. 10, pp. 33-55). CRM Documents. Bellaterra, Barcelona: Centre de Recerca Matemàtica.

Artigue, M., Bosch, M., \& Gascón, J. (2011b). Research praxeologies and networking theories. En M. Pytlak, T. Rowland, \& E. Swoboda (Eds.), Proceedings of the Seventh Congress of the European Society for Research in Mathematics Education CERME7 (pp. 2381-2390). Rzeszów, Polonia: University of Rzeszów.

Artigue, M., Bosch, M., Gascón, J., \& Lenfant, A. (2010). Research problems emerging from a teaching episode: a dialogue between TDS and ATD. En V. Durand-Guerrier, S. Soury-Lavergne, \& F. Arzarello (Eds.), Proceedings of the Sixth Congress of the European Society for Research in Mathematics Education (pp. 1535-1544). Lyon, Francia: INRP. www.inrp.fr/editions/cerme6

Arzarello, F., Bosch, M., Gascón, J., \& Sabena, C. (2008). The ostensive dimension through the lenses of two didactic approaches. ZDM - Mathematics Education, 40, 179 -188.

Bauersfeld, H. \& Skowronek, H. (1976). Research related to the mathematical learning process. En Athen, \& Kunle (Eds.), Proceedings of the Third International Congress on Mathematical Education (pp. 231-245). RFA:Universität Karlsrule, Zentralbatt für didaktik der Mathematik Karlsrule.

Bikner-Ahsbahs, A., Dreyfus, T., Kidron, I., Arzarello, F., Radford, L., Artigue, M., \& Sabena, C. (2010). Networking of theories in mathematics education. En M. M. F. Pinto, \& T. F. Kawasaki (Eds.), Proceedings of PME 34 (vol. 1, pp. 145-175).

Bolea, P., Bosch, M., García, J., Gascón, J., Ruiz Higueras, L., \& Sierra, T. A. (2005). Analyse de «La mesure en CM1» d'aprés la TAD. En M. H. Salin, P. Clanché, \& B. Sarrazy (Eds.), Sur la Théorie des Situations Didactiques (pp. 153-166). Grenoble, Francia: La Pensée sauvage 
Bolea, P. (2003). El proceso de algebrización de organizaciones matemáticas escolares. Monografía del Seminario Matemático García de Galdeano, 29. Zaragoza, España: Departamento de Matemáticas de la Universidad de Zaragoza.

Bolea, P., Bosch, M., \& Gascón, J. (2001a). Cómo se construyen los problemas en Didáctica de las Matemáticas. Educación Matemática, 13(3), 22-63.

Bolea, P., Bosch, M., \& Gascón, J. (2001b). La transposición didáctica de organizaciones matemáticas en proceso de algebrización. El caso de la proporcionalidad. Recherches en Didactique des Mathématiques, 21(3), 247-304.

Bolea, P., Bosch, M., \& Gascón, J. (2004). Why is modelling not included in the teaching of algebra at secondary school? Quaderni di Ricerca in Didattica, 14, 125-133.

Bosch, M. (2012). Recorridos de Investigación en Didáctica de las Matemáticas: el grupo TAD. En A. Estepa, A. Contreras, J. Deulofeu, M.C. Penalva, F. J. García, \& L. Ordóñez (Eds.), Investigación en Educación Matemática XVI (pp.23-47). Jaén, España: SEIEM.

Bosch, M., \& Gascón, J. (2006). 25 years of the Didactic Transposition. Bulletin of the International Commission on Mathematical Instruction 58, 51-65.

Bosch, M., Gascón, J., Ruiz Olarría, A., Artaud, M., Bronner, A., Chevallard, Y., Cirade, G., Ladage, C., \& Larguier, M. (Eds.) (2011). Un panorama de la TAD. CRM Documents, vol. 10. Bellaterra, Barcelona: Centre de Recerca Matemàtica.

Bronner, A., Larguier, M., Artaud, M., Bosch, M., Chevallard, Y., Cirade, G., \& Ladage, C. (Eds.) (2010). Diffuser les mathématiques (et les autres savoirs) comme outils de connaissance et d'action. Montpellier, Francia: IUFM de l'Académie de Montpellier.

Brousseau, G. (1986). Fondements et méthodes de la didactiques des mathématiques. Recherches en didactique des Mathématiques, 7(2), 33-115.

Brousseau, G. (1994). Problèmes et résultats de Didactique des Mathématiques. Washington: ICMI Study 94.

Brousseau, G. (1996). La didàctica en la formació del professorat, Butlletí de la Societat Catalana de Matemàtiques, Institut d'Estudis Catalans, 11(1), 33-45. (Conferencia pronunciada en las Segones Jornades de Matemàtiques i Didàctica de les Matemàtiques, celebradas en febrero de 1993 en la Universitat Autònoma de Barcelona).

Brousseau, G. (1997). Theory of Didactical Situations in Mathematics. Didactique des mathématiques, 1970 - 1990. En N. Balacheff, R. Sutherland, \& V. Warfield (Eds.). Dordrecht: Kluwer Academic Publishers.

Brousseau, G. (1998). Théorie des situations didactiques: Didactique des mathématiques 1970-1990. En N. Balacheff, M. Cooper, R. Sutherland, \& V. Warfield (Eds.). Grenoble: La pensée sauvage.

Brousseau, G. (2007). Iniciación al estudio de la teoría de las situaciones didácticas, [Traducción de Dilma Fregona]. Buenos Aires: Libros del Zorzal.

Chevallard, Y. (1985). La transposition didactique. Du savoir savant au savoir eneigné. Grenoble: La pensée Sauvage.

Chevallard Y., Bosch M., \& Gascón J. (1997). Estudiar matemáticas. El eslabón perdido entre la enseñanza y el aprendizaje. Barcelona: ICE/Horsori.

D'Amore, B., \& Godino, J. D. (2007) El enfoque ontosemiótico como un desarrollo de la teoría antropológica en Didáctica de la Matemática. Revista Latinoamericana de Investigación en Matemática Educativa, 10(2), 191-218.

Drijvers, P. Godino, J. D., Font, V., \& Trouche, L. (2012). One episode, two lenses. A reflective analysis of student learning with computer 
algebra from instrumental and onto-semiotic perspectives. Educational Studies in Mathematics. doi: 10.1007/s10649-012-9416-8.

Font, V., Godino, J. D., \& D'Amore, B. (2007). Enfoque ontosemiótico de las representaciones en educación matemática. An onto-semiotic approach to representations in mathematics education. For the Learning of Mathematics, 27(2), 2-7.

García, F. J., \& Ruiz-Higueras, L. (2010). Exploring the use of theoretical frameworks for modelling-oriented instructional design. En V. Durand-Guerrier, S. Soury-Lavergne, \& F. Arzarello (Eds.), Proceedings of the Sixth Congress of the European Society for Research in Mathematics Education (pp. 2166-2175). Lyon, Francia: Service des Publications, Institut National de Recherche Pédagogique.

García, F. J., \& Ruiz-Higueras, L. (2011). Modifying teachers' practices: the case of a European Training Course on Modelling and Applications. En G. Kaiser, W. Blum, R. Borromeo-Ferri, \& G. Stillman (Eds.), Trends in Teaching and Learning of Mathematical Modelling (pp. 569-578). Dordrecth, Países Bajos: Springer.

García, F. J., \& Wake, G. (2010). Estableciendo diálogos entre diferentes marcos teóricos: de los procesos narrativos a la teoría antropológica de lo didáctico. Investigación en Educación Matemática, 14, 315-326.

Gascón, J. (1993). Desarrollo del conocimiento matemático y análisis didáctico: Del Patrón Análisis-Síntesis a la génesis del lenguaje algebraico. Recherches en didactique des mathématiques, 13(3), 295-332.

Gascón, J. (1999). La naturaleza prealgebraica de la matemática escolar. Educación matemática, 11(1), 77-88.

Gascón, J. (1994-1995). Un nouveau modèle de l'algèbre élémentaire comme alternative à l'« arithmétique généralisée ». Petit $x, 37,43-63$.

Gascón, J. (1998). Evolución de la didáctica de las matemáticas como disciplina científica. Recherches en Didactique des Mathématiques, 18(1), 7-34.

Gascón, J. (2001). Incidencia del modelo epistemológico de las matemáticas sobre las prácticas docentes. Revista Latinoamericana de Investigación en Matemática Educativa RELIME, 4(2), 129-159.

Gascón, J. (2002). El problema de la Educación Matemática y la doble ruptura de la Didáctica de las Matemáticas. Gaceta de la Real Sociedad Matemática Española, 5(3), 673-698.

Gascón, J. (2003). From the cognitive to the epistemological programme in the didactics of mathematics: two incommensurable scientific research programmes, For the learning of mathematics, 23(2), 44-55.

Gascón, J. (2011). ¿Qué problema se plantea el enfoque por competencias? Un análisis desde la Teoría Antropológico de lo Didáctico. Recherches en Didactique des Mathématiques, 31(1), 9-50.

Gascón, J. (2012). La revolución brousseauniana como razón de ser del grupo DMDC. En A. Estepa, A. Contreras, J. Deulofeu, M.C. Penalva, F. J. García, \& L. Ordóñez (Eds.), Investigación en Educación Matemática XVI (pp.15-22). Jaén, España: SEIEM.

Godino, J. D. (2012). Origen y aportaciones de la perspectiva ontosemiótica de investigación en Didáctica de la Matemática. En A. Estepa, A. Contreras, J. Deulofeu, M.C. Penalva, F. J. García, \& L. Ordóñez (Eds.), Investigación en Educación Matemática XVI (pp.4968). Jaén, España: SEIEM.

Godino, J. D., Font, V., Contreras, A., \& Wilhelmi, M. R. (2006). Una visión de la didáctica francesa desde el enfoque ontosemiótico de la 
cognición e instrucción matemática. Revista Latinoamerica de Investigación en Matemática Educativa, 9(1), 117-150.

Godino, J. D. et al. (2012). Métodos de investigación en las ponencias y comunicaciones presentadas en los simposios de la SEIEM. Avances de Investigación en Educación Matemática, 2, 29 - 52.

Lakatos, I. (1978). The Methodology of Scientific Research Programmes: Philosophical Papers, Vol. 1. Cambridge: Cambridge University Press.

Malara, N. A. (1997). An International View on Didactics of Mathematics as a Scientific Discipline. Modena, Italia: University of Modena.

Prediguer, S., Arzarello, F., Bosch, M., \& Lenfant, A. (2008). Comparing, combining, coordinating - netowrking strategies for connecting theoretical approaches. ZDM Mathematics Education, 40(2), 163-164.

Rodríguez, E., Bosch, M., \& Gascón, J. (2008). A networking method to compare theories: metacognition in problem solving reformulated within the anthropological theory of the didactic. ZDM Mathematics Education, 40, 287-301.

Ruiz-Higueras, L., Orús, P., Godino, J.D., \& Gascón, J. (2001). Perspectiva de la investigación del grupo didáctica de las matemáticas como disciplina científica. En L. Contreras et al. (Eds.), Cuarto Simposio de la Sociedad Española de Investigación en Educación Matemática (collectanea ํ54, pp. 175-182). Huelva, España: Universidad de Huelva.

Ruiz-Higueras, L. Estepa, A., \& García, F.J. (2007). Sociedad, escuela y matemáticas. Aportaciones de la Teoría Antropológica de lo Didáctico (TAD). Jaén, España: Publicaciones de la Universidad de Jaén.

Ruiz-Munzón, N. (2010). La introducción del álgebra elemental y su desarrollo hacia la modelización funcional (Tesis doctoral). Universitat Autònoma de Barcelona, Barcelona, España.

Ruiz-Munzón, N., Bosch, M., \& Gascón, J. (2010). La algebrización de los programas de cálculo aritmético y la introducción del álgebra en secundaria. En M. M. Moreno, A. Estrada, J. Carrillo, \& T. A. Sierra (Eds.), Investigación en Educación Matemática XIV (pp. 545-556). Lleida, España: SEIEM.

Ruiz-Munzón, N., Bosch, M., \& Gascón, J. (2011). Un modelo epistemológico de referencia del algebra como instrumento de modelización. En M. Bosch, J. Gascón, A. Ruiz Olarría, M. Artaud, A. Bronner, Y. Chevallard, G. Cirade, C. Ladage, \& M. Larguier (Eds.), Un panorama de la TAD (vol. 10, pp. 743-765). CRM Documents. Bellaterra, Barcelona: Centre de Recerca Matemàtica.

Ruiz-Munzón, N., Matheron, Y., Bosch, M., \& Gascón, J. (en prensa). Autour de l'algèbre: les entiers relatifs et la modélisation algébrico-fonctionnelle. Recherches en Didactique des Mathématiques.

Shulman, L.S. (1986). Those who understand: Knowledge growth in teaching. Educational Researcher, 15(2), 4-14.

Shulman, L.S. (1987). Knowledge and teaching: Foundations of a new reform. Harward Educational Review, 57(1), 1-22.

Sierra, T.A., Bosch, M. y Gascón, J. (2012). La formación matemático-didáctica del maestro de educación infantil: El caso de "enseñar a contar". Revista de Educación, 357, 231256.

Thurston, W. P. (1994). On proof and progress in mathematics, Bulletin of the American Mathematical Society, 30(2), 161-177. 
Trigueros, M., Bosch, M., \& Gascón, J. (2011). Tres modalidades de diálogo entre APOS y TAD. En M. Bosch, J. Gascón, A. Ruiz Olarría, M. Artaud, A. Bronner, Y. Chevallard, G. Cirade, C. Ladage, \& M. Larguier (Eds.), Un panorama de la TAD (vol. 10, pp. 77116). CRM Documents. Bellaterra, Barcelona: Centre de Recerca Matemàtica.

Weber, M. (2009). La "objetividad del conocimiento en la ciencia social y en la política social [Traducción de J. Abellán]. Madrid: Alianza Editorial (Edición original: 1904).

Wilhelmi, M. R., Font, V., \& Godino, J. D. (2005). Bases empíricas de modelos teóricos en didáctica de las matemáticas: Reflexiones sobre la Teoría de Situaciones Didácticas y el Enfoque Ontológico y Semiótico. Traducción de "Bases empiriques de modèles théoriques en didactique des mathématiques: réflexions sur la théorie de situations didactiques et le point de vue ontologique et sémiotique". Bordeaux, France : Colloque International Didactiques: quelles references epistemologiques?, "Association Francophone Internationale de Recherche Scientifique en Education (AFIRSE)”, IUFM d'Aquitaine.

\section{Referencia del autor}

Josep Gascón, departamento de Matemáticas, Universitat Autònoma de Barcelona (España). gascon@mat.uab.cat 


\section{A revolução brousseauniana como razão de ser do grupo Didática da Matemática como uma Disciplina Científica}

Josep Gascón, Universitat Autònoma de Barcelona (España)

This work presents an interpretation of the history of a small community of research in didactics of mathematics which has culminated in the group Didactics of Mathematics as a Scientific Discipline (DMDC) of the Spanish Society for Research in Mathematics Education (SEIEM). This community started in 1991 as an interuniversity seminar and evolve until 1998 giving rise to the DMDC.

The researchers integrating this group mainly share a specific way of interpreting didactics, with the nuances and variations provided by the different approaches and scientific interests assumed. This interpretation is inspired in what we can call the "Brousseaunian revolution" of didactic of mathematics, thus making some of the basic assumptions of this revolution appear as the rationale of the group DMDC.

Two of the basic assumptions or principles that characterise the Brousseaunian revolution and have been leading the development of the Theory of didactic situations (TDS) can be formulated as follows: (1) The TDS has the ambition to build a relatively autonomous science of didactics, with its own object of study, where the notion of "didactic phenomenon" plays a central role; (2) The TDS considers mathematical activity as the primary object of study of didactics and as a new "entrance" of didactic analysis. This is why it can be said that the TDS inaugurates a new programme of research in didactics of mathematics, the epistemological programme.

As a fundamental consequence of both principles appears the necessity to question the common ways of describing and interpreting "mathematical knowledge" and the consequent necessity to build, within the didactics, specific epistemological models (of the different mathematical domains) to approach the didactic problems where this knowledge is involved. This is probably the most important principle, which in a sense contains the previous ones, and it characterises the raising of didactics of mathematics as an experimental epistemology.

In coherence with these considerations, we propose to characterise the approaches or theories belonging to the epistemological programme as those questioning the epistemological models of mathematics prevailing in different institutions (school ones, for instance) and, what is more important, as those that explicitly elaborate alternative epistemological models of the different mathematical domains and use them as a reference system to formulate and approach didactic problems. 